\title{
Las analíticas de aprendizaje en el Ecuador: Un análisis inicial basado en el mapeo sistemático de los trabajos de graduación
}

\begin{abstract}
Learning analytics in Ecuador: An initial analysis based on the systematic mapping of graduation works
\end{abstract}

Rubén Antonio Pazmiño Maji. ${ }^{1}$, Jorge Rigoberto López Ortega. ${ }^{2}$, Miguel Ángel Conde González. ${ }^{3}$ \& Francisco José García Peñalvo. ${ }^{4}$

\begin{abstract}
Learning Analytics are the collection, analysis and visualization of data from the learning process and its environment. The use of Learning Analytics provides the opportunity to build models that allow analyzing and strengthening the learning process at the middle and higher educational level. In the last five years (2014-2018), the Scopus bibliographic database shows 2180 documents related to Learning Analytics; this shows the importance and growing interest in this line of research. This research uses the Mapping Review of Literature to answer five questions about undergraduate and postgraduate degree works in the area of Learning Analytics by Ecuadorian authors. The study was carried out in the last five years (2014-2018), the digital repositories Senescyt and rraae were used as sources of information. The initial searches resulted in thirteen documents on Learning Analytics produced by Ecuadorian authors. The application of the inclusion, exclusion and quality criteria decreased the number of documents; three graduation works are finally used. This work contributes to showing the Higher Education Institutions, educational levels, keywords, years and authors of the undergraduate and postgraduate graduation papers on Learning Analytics in Ecuador. The answers to the research questions posed will allow researchers to determine the

1 Escuela Superior Politécnica de Chimborazo, Facultad de Ciencias, Riobamba, Ecuador, rpazmino@espoch.edu.ec

2 Escuela Superior Politécnica de Chimborazo, Facultad de Recursos Naturales, Riobamba, Ecuador, jorge.lopezo@espoch.edu.ec

3 University of León, León, Spain, miguel.conde@unileon.es

4 University of Salamanca, Salamanca, Spain, fgarcia@usal.es
\end{abstract}


characteristics of undergraduate and postgraduate degree works on Learning Analytics that have been made in Ecuador in the last five years. This research is fully replicable if the same sources of information and the same objectives are used.

Keywords: Learning Analytics, Ecuador, Systematic Mapping, graduation work.

\section{Resumen}

Las Analíticas de Aprendizaje son la recopilación, análisis y visualización de datos del proceso de aprendizaje y su entorno. La utilización de las Analíticas de Aprendizaje provee la oportunidad para construir modelos que permitan analizar y fortalecer el proceso de aprendizaje a nivel educativo medio y superior. En los últimos 5 años (2014-2018), la base de datos bibliográfica Scopus muestra 2180 documentos relacionados con las Analíticas de Aprendizaje, esto evidencia la importancia y el interés cada vez más creciente en esta línea de investigación. Esta investigación utiliza el Mapeo Sistemático de Literatura para responder cinco preguntas sobre los Trabajos de Graduación de pregrado y postgrado en el área de las Analíticas de Aprendizaje de autores ecuatorianos. El estudio se realizó en los últimos 5 años (2014-2018), se utilizaron como fuentes de información los repositorios digitales Senescyt y rraae. Las búsquedas iniciales dieron como resultado 13 Trabajos de Graduación sobre Analíticas de Aprendizaje producidos por autores ecuatorianos. La aplicación de los criterios de inclusión, exclusión y calidad disminuyó el número de documentos, finalmente se utilizaron tres Trabajos de Graduación. Este trabajo contribuye mostrando las instituciones de educación superior, los niveles educativos, las palabras clave, los años y los autores de los Trabajos de Graduación de pregrado y posgrado en Learning Analytics en Ecuador. Las respuestas a las preguntas de investigación planteadas permitirán a los investigadores determinar las características de los Trabajos de Graduación sobre Learning Analytics que se han realizado en Ecuador. Esta investigación es plenamente reproducible si se utilizan las mismas fuentes de información y los mismos objetivos.

Palabras claves: Analíticas de Aprendizaje, Ecuador, Mapeo Sistemático, Trabajos de Graduación.

\section{Introducción}

Los recursos en esta página proporcionan una introducción al tema de las aplicaciones de software de análisis de aprendizaje en la educación superior. Al momento de presentar este artículo científico no existe ninguna documentación que muestre el estado actual de las Analíticas de Aprendizaje en el Ecuador, los artículos más cercanos son los titulados "An Initial Review of Learning Analytics in Latin America” (Dos Santos, Cechinel, Nunes, \& Ochoa, 2017) y "Learning Analytics in Latin America present an opportunity not to be missed" (Ochoa, 2019) ambos escritos por el ecuatoriano Xavier Ochoa.

Las Analíticas de Aprendizaje se definen como "la medición, recopilación, análisis e informe de datos sobre los alumnos y su contexto, con el fin de comprender y optimizar el aprendizaje 
y los entornos en los que ocurre" (Siemens \& Long, 2011) o también como "una aplicación educativa de análisis web dirigida a la elaboración de perfiles de los alumnos, un proceso de recopilación y análisis de detalles de las interacciones individuales de los estudiantes en las actividades de aprendizaje en línea" (Johnson et al., 2016). La llegada del aprendizaje en línea ha permitido rastrear el camino del estudiante hacia la comprensión y el dominio de los conocimientos de un curso. Este tipo de seguimiento conocido como "Analíticas de Aprendizaje" es un tema candente entre los educadores, la pregunta que todos debaten es ¿Cómo se pueden aprovechar los datos para mejorar el diseño del curso y el aprendizaje? Los intentos de imaginar el futuro de la Educación Superior a menudo enfatizan en las nuevas tecnologías de la información y comunicación tales como: dispositivos informáticos ubicuos (Feliz González, 2017), diseños de aulas flexibles y pantallas visuales innovadoras. Basar las decisiones en datos y evidencias parece sorprendentemente obvio, la toma de decisiones basada en datos mejora el rendimiento y la productividad de las organizaciones educativas.

La historia de la educación en el Ecuador ha llevado un camino ascendente, con tropiezos ha logrado vencer serias limitaciones provenientes de la pobreza, de los prejuicios y de la ineptitud. En 1928 se fundó la Facultad de Filosofía y Letras en la Universidad Central de Quito y dentro de ella el Instituto de Pedagogía. Desde entonces ha habido serios esfuerzos por mejorar la formación profesional de los docentes, sin olvidar el recurrente problema de la politización de la educación, agravado por el escaso presupuesto del ministerio pertinente.

La educación particular pasó por momentos muy difíciles desde inicios del siglo hasta el reconocimiento de ese derecho en 1946, pero el proceso fue lento. Junto a excelentes centros no han faltado aquellos mediocres. Sin embargo, dentro de esta área de la educación no solo han surgido innovaciones positivas sino también proyectos en beneficio de los sectores menos favorecidos. Ha sido una constante a lo largo del siglo XX el sucederse de "reformas pedagógicas y curriculares", a veces más fundamentadas, a veces menos. Además, a partir de mediados de ese siglo, gracias a las rentas del banano y luego del petróleo, ha aumentado sustancialmente el número y la calidad de los establecimientos educativos (Freile, 2015).

Rraae es la Red de Repositorios de Acceso Abierto de Ecuador es el motor de búsqueda nacional, su propósito es facilitar la gestión, descentralización, organización, preservación e interoperabilidad de contenido digital de acceso abierto, generado por las instituciones de la comunidad académica y científica del Ecuador. Se promueve la visibilidad de la producción de conocimiento distribuido en los diferentes repositorios institucionales a nivel nacional. El rraae es el nodo nacional que forma parte de la Red Federal de Repositorios Institucionales de Publicaciones Científicas de América Latina («Rraae», s. f.).

Actualmente el Ecuador cuenta con 61 Instituciones de Educación Superior («Portal Ciudadano SNIESE», s. f.) de las cuales 32 se encuentran inscritas en la rraae (ver Tabla 1). 
Tabla 1: Todas las Universidades del Ecuador y aquellas inscritas a la red rraae

\begin{tabular}{|c|c|c|}
\hline No. & Universidades Ecuatorianas & RRAAE \\
\hline 1 & ESCUELA POLITECNICA NACIONAL & $\mathrm{Si}$ \\
\hline 2 & ESCUELA SUPERIOR POLITECNICA AGROPECUARIA DE MANABI & $\mathrm{Si}$ \\
\hline 3 & ESCUELA SUPERIOR POLITECNICA DE CHIMBORAZO & $\mathrm{Si}$ \\
\hline 4 & ESCUELA SUPERIOR POLITECNICA DEL LITORAL & $\mathrm{Si}$ \\
\hline 5 & FACULTAD LATINOAMERICANA DE CIENCIAS SOCIALES & \\
\hline 6 & INSTITUCIÓN EXTRANJERA & \\
\hline 7 & INSTITUTO DE ALTOS ESTUDIOS NACIONALES & $\mathrm{Si}$ \\
\hline 8 & PONTIFICIA UNIVERSIDAD CATOLICA DEL ECUADOR (Sedes Quito y Ambato) & $\mathrm{Si}$ \\
\hline 9 & UNIVERSIDAD AGRARIA DEL ECUADOR & \\
\hline 10 & UNIVERSIDAD ANDINA SIMON BOLIVAR & $\mathrm{Si}$ \\
\hline 11 & UNIVERSIDAD CASA GRANDE & $\mathrm{Si}$ \\
\hline 12 & UNIVERSIDAD CATOLICA DE CUENCA & \\
\hline 13 & UNIVERSIDAD CATOLICA DE SANTIAGO DE GUAYAQUIL & $\mathrm{Si}$ \\
\hline 14 & UNIVERSIDAD CENTRAL DEL ECUADOR & $\mathrm{Si}$ \\
\hline 15 & UNIVERSIDAD DE CUENCA & $\mathrm{Si}$ \\
\hline 16 & UNIVERSIDAD DE ESPECIALIDADES TURISTICAS & \\
\hline 17 & UNIVERSIDAD DE GUAYAQUIL & $\mathrm{Si}$ \\
\hline 18 & UNIVERSIDAD DE INVESTIGACION DE TECNOLOGIA EXPERIMENTAL YACHAY & $\mathrm{Si}$ \\
\hline 19 & UNIVERSIDAD DE LAS AMERICAS & $\mathrm{Si}$ \\
\hline 20 & UNIVERSIDAD DE LAS ARTES & $\mathrm{Si}$ \\
\hline 21 & UNIVERSIDAD DE LAS FUERZAS ARMADAS (ESPE) & $\mathrm{Si}$ \\
\hline 22 & UNIVERSIDAD DE LOS HEMISFERIOS & \\
\hline 23 & UNIVERSIDAD DE OTAVALO & $\mathrm{Si}$ \\
\hline 24 & UNIVERSIDAD DEL AZUAY & $\mathrm{Si}$ \\
\hline 25 & UNIVERSIDAD DEL PACIFICO ESCUELA DE NEGOCIOS & \\
\hline 26 & UNIVERSIDAD DEL RÍO & \\
\hline 27 & UNIVERSIDAD ESTATAL AMAZONICA & $\mathrm{Si}$ \\
\hline 28 & UNIVERSIDAD ESTATAL DE BOLIVAR & $\mathrm{Si}$ \\
\hline 29 & UNIVERSIDAD ESTATAL DE MILAGRO & $\mathrm{Si}$ \\
\hline 30 & UNIVERSIDAD ESTATAL DEL SUR DE MANABI & \\
\hline 31 & UNIVERSIDAD ESTATAL PENINSULA DE SANTA ELENA & $\mathrm{Si}$ \\
\hline 32 & UNIVERSIDAD IBEROAMERICANA DEL ECUADOR & \\
\hline 33 & UNIVERSIDAD INTERNACIONAL DEL ECUADOR & $\mathrm{Si}$ \\
\hline 34 & UNIVERSIDAD LAICA ELOY ALFARO DE MANABI & \\
\hline 35 & UNIVERSIDAD LAICA VICENTE ROCAFUERTE DE GUAYAQUIL & \\
\hline 36 & UNIVERSIDAD METROPOLITANA & \\
\hline 37 & UNIVERSIDAD NACIONAL DE CHIMBORAZO & $\mathrm{Si}$ \\
\hline 38 & UNIVERSIDAD NACIONAL DE EDUCACION UNAE & \\
\hline 39 & UNIVERSIDAD NACIONAL DE LOJA & $\mathrm{Si}$ \\
\hline 40 & UNIVERSIDAD PARTICULAR DE ESPECIALIDADES ESPIRITU SANTO & \\
\hline 41 & UNIVERSIDAD PARTICULAR INTERNACIONAL SEK & \\
\hline 42 & UNIVERSIDAD PARTICULAR SAN GREGORIO DE PORTOVIEJO & \\
\hline
\end{tabular}


43 UNIVERSIDAD POLITECNICA ESTATAL DEL CARCHI Si

44 UNIVERSIDAD POLITECNICA SALESIANA Si

45 UNIVERSIDAD REGIONAL AMAZONICA IKIAM

46 UNIVERSIDAD REGIONAL AUTONOMA DE LOS ANDES Si

47 UNIVERSIDAD SAN FRANCISCO DE QUITO Si

48 UNIVERSIDAD TECNICA DE AMBATO $\quad \mathrm{Si}$

49 UNIVERSIDAD TECNICA DE BABAHOYO

50 UNIVERSIDAD TECNICA DE COTOPAXI

51 UNIVERSIDAD TECNICA DE MACHALA Si

52 UNIVERSIDAD TECNICA DE MANABI

53 UNIVERSIDAD TECNICA DEL NORTE $\quad \mathrm{Si}$

54 UNIVERSIDAD TECNICA ESTATAL DE QUEVEDO Si

55 UNIVERSIDAD TECNICA LUIS VARGAS TORRES DE ESMERALDAS

56 UNIVERSIDAD TECNICA PARTICULAR DE LOJA Si

57 UNIVERSIDAD TECNOLOGICA ECOTEC

58 UNIVERSIDAD TECNOLOGICA EMPRESARIAL DE GUAYAQUIL

59 UNIVERSIDAD TECNOLOGICA INDOAMERICA Si

60 UNIVERSIDAD TECNOLOGICA ISRAEL

61 UNIVERSIDAD UTE $\quad \mathrm{Si}$

TOTAL 36

En este estudio se han considerado un total de 199965 Trabajos de Graduación correspondientes al 59\% de Universidades ecuatorianas (36 en total), repartidas como se muestra en la Tabla 2.

Tabla 2: Distribución de los 225717 Trabajos de Graduación considerados

FUENTE: («Rraae», s. f.)

\begin{tabular}{lcc}
\hline \multicolumn{1}{c}{ Trabajo de Graduación } & Número \\
\hline Tesis de pregrado & 172067 \\
\hline Tesis de Maestría & 27486 \\
\hline Tesis de Doctorado & & 412 \\
\hline & TOTAL & $\mathbf{1 9 9 9 6 5}$ \\
\hline
\end{tabular}

En la Figura 1, se muestran las provincias, las Universidades y sus respectivos repositorios digitales desde donde se cosecha la información que alimenta frecuentemente a la rraae. 


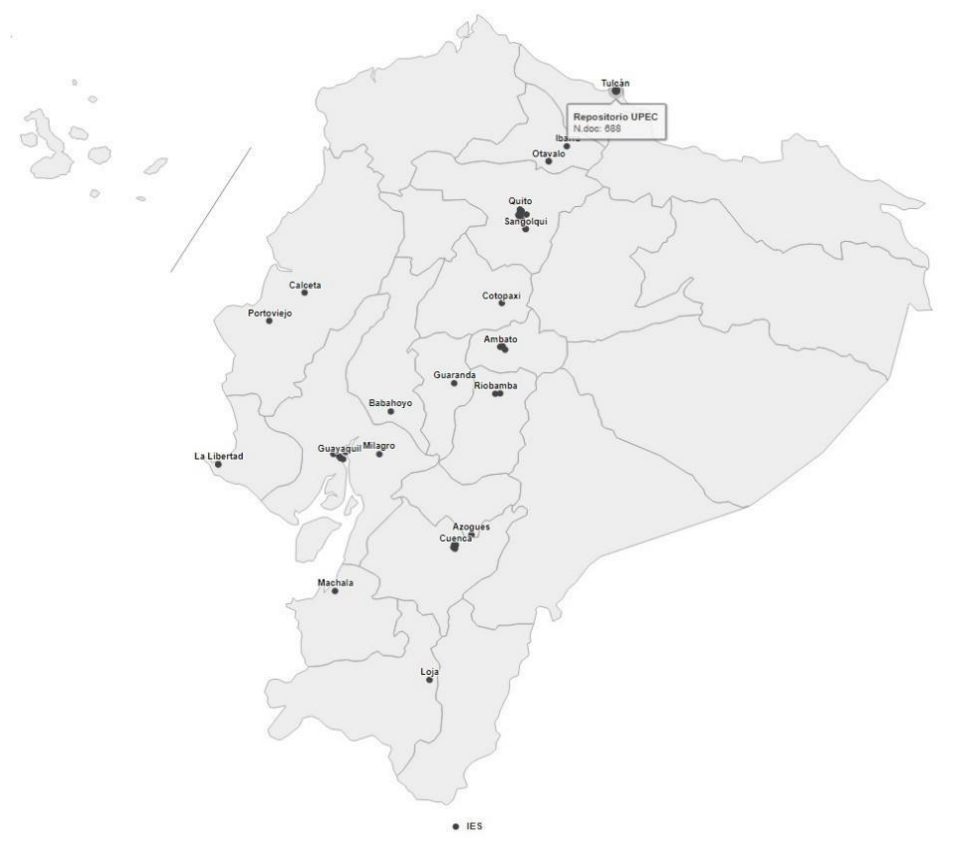

Figura 1: Repositorio Universitarios cosechados por rraae por provincia FUENTE: («Estadísticas», s. f.)

Los Trabajos de Graduación de pregrado se guían por el Reglamento de Régimen Académico elaborado por EL CONSEJO DE EDUCACIÓN SUPERIOR («CES - Consejo de Educación Superior | Ecuador», s. f.) con resolución RPC-SO-08-No.111-2019 («Buscar en Gaceta Oficial |CES - Consejo de Educación Superior|Ecuador», s. f.), que en sus artículos número 32 y 33 respectivamente indican: “Artículo 32.- Diseño, acceso y aprobación de la unidad de integración curricular del tercer nivel.- Cada IES diseñará la unidad de integración curricular, estableciendo su estructura, contenidos y parámetros para el correspondiente desarrollo y evaluación. Para acceder a la unidad de integración curricular, es necesario haber completado las horas y/o créditos mínimos establecidos por la IES, así como cualquier otro requisito establecido en su normativa interna. Su aprobación se realizará a través de las siguientes opciones: a) Desarrollo de un trabajo de integración curricular; o, b) La aprobación de un examen de carácter complexivo, mediante el cual el estudiante deberá demostrar el manejo integral de los conocimientos adquiridos a lo largo de su formación. Las IES podrán ofrecer una o ambas opciones de los literales anteriores para la aprobación de la unidad de integración curricular. El desarrollo de esta unidad deberá asegurar la evaluación y calificación individual, con independencia de los mecanismos de trabajo implementados. Una vez aprobada la unidad de integración curricular, su calificación deberá ser registrada de manera inmediata. Se podrá emitir el título respectivo únicamente cuando el estudiante apruebe todos los requisitos académicos y administrativos establecidos en la resolución de aprobación de la carrera o programa, lo que constará en el acta consolidada de finalización de estudios, en conformidad con el artículo 101 de este Reglamento. Artículo 33.- Reprobación de unidad de integración curricular,- Un estudiante podrá reprobar hasta 
dos (2) veces la unidad de integración curricular, y solicitar autorización para cursarla por tercera (3) ocasión mediante los mecanismos definidos por la IES. En caso que la IES ofrezca las dos (2) opciones de aprobación de la unidad de integración curricular, establecidos en el artículo precedente, podrá cambiarse una única vez de opción mediante el proceso que establezca la IES".

Los Trabajos de Graduación de postgrado se guían por el Reglamento de Régimen Académico elaborado por EL CONSEJO DE EDUCACIÓN SUPERIOR («CES - Consejo de Educación Superior | Ecuador», s. f.) con resolución RPC-SO-08-No.111-2019 («Buscar en Gaceta Oficial | CES - Consejo de Educación Superior | Ecuador», s. f.), que en sus artículos número 34 y 35 respectivamente indican: "Artículo 34.- Unidades de organización curricular del cuarto nivel.- Un programa de posgrado deberá contar con las siguientes unidades: c) Unidad de titulación.- Valida las competencias profesionales, tecnológicas y/o investigativas para el abordase de situaciones, necesidades, problemas, dilemas o desafíos de la profesión y los contextos desde un enfoque reflexivo, investigativo, experimental, innovador, entre otros. Artículo 35.- Diseño, acceso y aprobación de unidad de titulación del cuarto nivel.- Cada IES diseñará su unidad de titulación de posgrado, estableciendo su estructura, contenidos y parámetros para su desarrollo y evaluación; distinguiendo la trayectoria de investigación y profesional, según lo indicado en el artículo 22 de este Reglamento. Para acceder a la unidad de titulación es necesario haber completado las horas y/o créditos mínimos establecidos por la IES. La aprobación implica haber completado y aprobado una (1) de las siguientes opciones: b) Posgrados con trayectoria de investigación: Tesis con componente de investigación básica y/o aplicada, con características de originalidad, relevancia y de impacto científico; que responda a las convenciones científicas del campo respectivo, pudiendo usar métodos propios de la disciplina o métodos multi e interdisciplinares. Las IES podrán establecer requisitos de artículos científicos enviados, aceptados y/o publicados para la titulación".

El propósito de un Mapeo Sistemático de Literatura (Jesson, Matheson, \& Lacey, 2011) es el de responder a las preguntas de investigación planteadas en forma clara y enfocada. También permite utilizando los criterios de inclusión que las preguntas contesten a hipótesis más sofisticadas (Pazmiño-Maji, García-Peñalvo, \& Conde-González, s. f.). Un Mapeo Sistemático de Literatura debe ser procesual, auditable y totalmente replicable, inicialmente se han aplicado a la revisión de literatura, pero pueden ser fácilmente utilizados en otros ámbitos (Pazmiño-Maji, García-Peñalvo, \& Conde-González, 2017) (Pazmiño-Maji, Valverde-Aguirre, García-Peñalvo, \& Conde-González, 2019) con mucho éxito. Las preguntas de investigación deben definirse al comienzo de su Mapeo Sistemático. Una pregunta de investigación bien formulada ayudará a determinar sus criterios de inclusión y exclusión, la creación de su estrategia de búsqueda, la recopilación de datos y la presentación de sus hallazgos. Una buena pregunta de investigación, permite encontrar rápidamente información válida (cumple con precisión los objetivos establecidos). Además, deben ser 
claras, inequívocas y estructuradas. Las preguntas de revisión generalmente tienen un enfoque preciso. Es importante formular las preguntas de investigación con cuidado para evitar perder estudios relevantes o recopilar un conjunto de resultados potencialmente sesgados (Kitchenham, Budgen, \& Brereton, 2016).

El método PICOC (población, intervención, comparación, salida (output) y contexto) es una herramienta útil para definir preguntas claras y específicas y desarrollar un protocolo de revisión. Además, es una herramienta útil para hacer preguntas y subpreguntas de investigación enfocadas (Coleman, s. f.). Así como PICOC hay otras metodologías para generar las preguntas de investigación tales como las Analíticas de Aprendizaje (PazmiñoMaji, Solis-Benavides, García-Peñalvo, \& Conde-González, 2019), PICOS y SPIDER (Methley, Campbell, Chew-Graham, McNally, \& Cheraghi-Sohi, 2014).

La Senescyt es la Secretaría de Educación Superior, Ciencia, Tecnología e innovación, para mantener la memoria de la educación superior, en el transcurso de los últimos años ha venido impulsando la utilización de su repositorio digital («Repositorio Digital Senescyt: Página de inicio», s. f.) basado en DSpace («DSpace: An Open Source Dynamic Digital Repository», s. f.). El repositorio digital de la Senescyt permite divulgar y fomentar la investigación en temas vinculados con la Educación Superior en el Ecuador y dar a conocer además todos los repositorios digitales de las Universidades del Ecuador como se muestra en la Figura 2.

\begin{tabular}{|l|l|}
\hline \multicolumn{2}{|c|}{ Otros Repositorios } \\
\hline Repositorio ANDINA & Repositorio EPN \\
\hline Repositorio ESPAM & Repositorio ESPE \\
\hline Repositorio ESPOL & Repositorio FLACSO \\
\hline Repositorio IAEN & Repositorio PUCE \\
\hline Repositorio SENESCYT & Repositorio UAZUAY \\
\hline Repositorio UCE & Repositorio UCUENCA \\
\hline Repositorio UDET & Repositorio UDLA \\
\hline Repositorio UEB & Repositorio UG \\
\hline Repositorio UIDE & Repositorio UISRAEL \\
\hline Repositorio ULEAM & Repositorio ULVR \\
\hline Repositorio UNACH & Repositorio UNEMI \\
\hline Repositorio UNIANDES & Repositorio UNL \\
\hline Repositorio UOTAVALO & Repositorio UPACIFICO \\
\hline Repositorio UPEC & Repositorio UPS \\
\hline Repositorio UPSE & Repositorio USFQ \\
\hline Repositorio UTA & Repositorio UTB \\
\hline Repositorio UTC & Repositorio UTE \\
\hline Repositorio UTEQ & Repositorio UTM \\
\hline Repositorio UTMACHALA & Repositorio UTN \\
\hline Repositorio UTPL & \multicolumn{1}{|l|}{} \\
\cline { 1 - 2 } & \\
\cline { 1 - 2 } &
\end{tabular}

Figura 2: Acceso a los Repositorios Digitales del Ecuador mediante Senescyt

En la sección de metodología se detalla el proceso de Mapeo Sistemático utilizado. En la sección de resultados, se responden a las cinco preguntas de investigación planteadas y en las conclusiones se sintetizan los resultados obtenidos. Las evidencias de lo expuesto en este 
artículo científico se pueden encontrar en el siguiente link https://liveespochedumy.sharepoint.com/:f:/g/personal/rpazmino_espoch_edu_ec/EmADu8 JGPkdOuH_ttoMTW zoBWw1kD_y38ThUGSF_JZOiCQ?e=ZVQLJp

\section{Metodología}

El objetivo de este Trabajo de investigación es el de realizar un acercamiento a las investigaciones sobre Analíticas de Aprendizaje en el Ecuador, utilizando para ello los Trabajos de Graduación tanto de pregrado como de postgrado en todas las Instituciones de Educación Superior Ecuatorianas. La metodología utilizada es el Mapeo Sistemático de Literatura, se elaboró un plan de ejecución que tuvo como objetivo responder a las siguientes cinco preguntas de investigación, que se muestran en la Tabla 3.

Tabla 3: Número y enunciado de las preguntas de investigación

\begin{tabular}{cl}
\hline No. & \multicolumn{1}{c}{ Enunciado de la Pregunta de Investigación } \\
\hline 1 & $\begin{array}{l}\text { ¿Cuáles Instituciones de Educación Superior tienen Trabajos de Graduación sobre } \\
\text { Analíticas de Aprendizaje? }\end{array}$ \\
\hline 2 & $\begin{array}{l}\text { ¿Cuáles Trabajos de Graduación sobre Analíticas de Aprendizaje son de pregrado o } \\
\text { postgrado? }\end{array}$ \\
\hline $3 \quad$ ¿Cuáles son las principales palabras clave de los Trabajos de Graduación sobre \\
& Analíticas de Aprendizaje en Instituciones de Educación Superior Ecuatorianas? \\
\hline 4 & $\begin{array}{l}\text { ¿Cuáles son los años de publicación y tendencia de los Trabajos de Graduación sobre } \\
\\
\text { Analíticas de Aprendizaje en Instituciones de Educación Superior Ecuatorianas? }\end{array}$ \\
\hline 5 & $\begin{array}{l}\text { ¿Cuáles son los autores y coautores de los Trabajos de Graduación sobre Analíticas } \\
\text { de Aprendizaje en Instituciones de Educación Superior Ecuatorianas? }\end{array}$ \\
\hline
\end{tabular}

El propósito de un Mapeo Sistemático de literatura es responder preguntas de investigación claras y enfocadas. PICOC es un método utilizado para describir los cinco elementos de las preguntas de investigación y facilitar su búsqueda, la Tabla 4, muestra la aplicación de este método:

Tabla 4: Método PICOC aplicado a las preguntas de investigación

\begin{tabular}{|c|c|c|c|c|c|}
\hline PICOC & RQ1 & RQ2 & RQ3 & RQ4 & RQ5 \\
\hline Población & \multicolumn{5}{|c|}{ Trabajos de Graduación de pregrado y postgrado en el Ecuador } \\
\hline Intervención & No Aplica & & & & \\
\hline Comparasión & No Aplica & & & & \\
\hline $\begin{array}{l}\text { Outcome } \\
\text { (Salida) }\end{array}$ & $\begin{array}{c}\text { Universidades } \\
\text { ecuatorianas }\end{array}$ & $\begin{array}{c}\text { Trabajos de } \\
\text { pregrado y } \\
\text { postgrado }\end{array}$ & $\begin{array}{c}\text { Principales } \\
\text { palabras } \\
\text { clave }\end{array}$ & $\begin{array}{c}\text { Años de } \\
\text { publicación }\end{array}$ & $\begin{array}{l}\text { Autores y } \\
\text { coautores }\end{array}$ \\
\hline Contexto & $\begin{array}{l}\text { Relacionados } \\
\text { ecuatorianos }\end{array}$ & $\begin{array}{l}\text { Analítica } \\
\text { esde } 2014 \text { has }\end{array}$ & $\begin{array}{l}\text { de aprendi } \\
\text { 2018) }\end{array}$ & ije realizados & por autores \\
\hline
\end{tabular}


El proceso gráfico de Mapeo Sistemático se observa en la Figura 3, y se muestra a continuación.

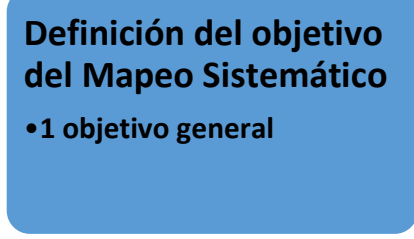

\section{Obtención de} Resultados

$\cdot 13$ Trabajos

\section{Aplicación de los} criterios de Inclusión, Exclusión y Calidad

-3 Trabajos de Grado
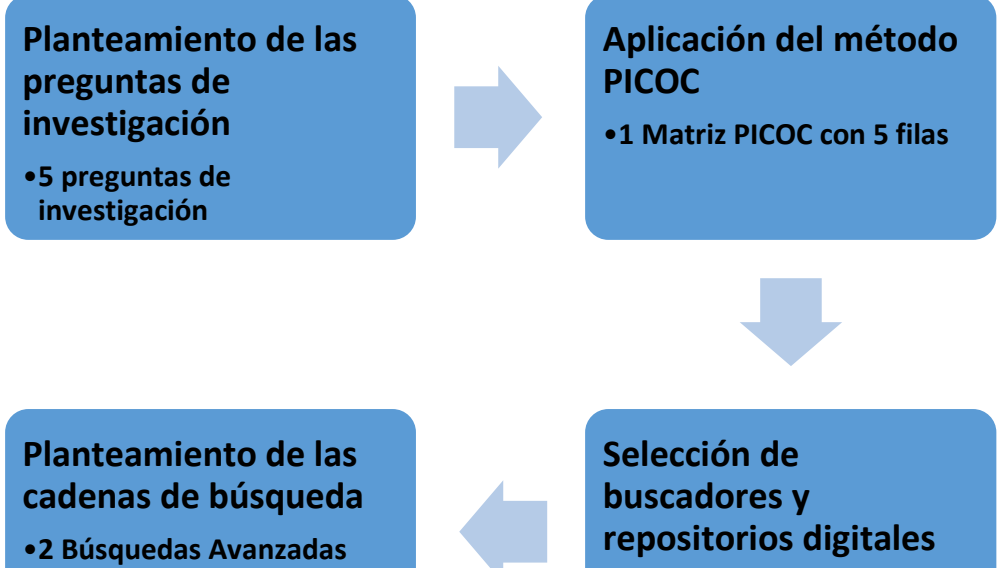

\section{Selección de} buscadores y repositorios digitales

-2 repositorios digitales

\section{Respuesta a las} preguntas de Investigación

-5 preguntas respondidas

\section{Elaboración de} conclusiones

-Conclusiones

Figura 3: Proceso de Mapeo Sistemático

Como se requieren los Trabajos de Pregrado y Postgrado del Ecuador se ha utilizado el repositorio digital de recursos abiertos del Ecuador rraae y Senescyt. Se utilizó una cadena de búsqueda avanzada (Hausner \& Waffenschmidt, 2016) con las características que se muestran en la Figura 4. 
Advanced Search

\begin{tabular}{|c|c|c|c|c|c|c|}
\hline \multirow[t]{4}{*}{ Search for: } & "Leaming Analytics" & All Fields & v & x & Match: & \\
\hline & & All Fields & $\checkmark$ & * & \multirow[t]{3}{*}{ ALL Terms } & \multirow[t]{3}{*}{$\nabla$} \\
\hline & & All Fields & 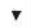 & x & & \\
\hline & 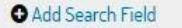 & & & & & \\
\hline
\end{tabular}

- Add Search Group

Limit To

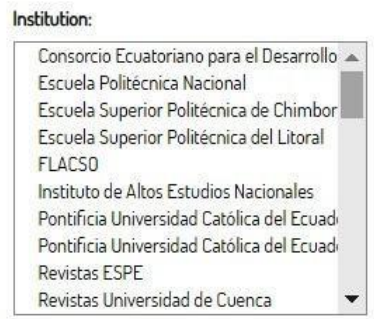

Format:

\section{Article}

Bachelor Thesis

Doctoral Thesis

Libro

Master Thesis

Objeto de conferencia

Revistas Universidad de Cuenca

Year of Publication

$\begin{array}{cc}\text { From: } & \text { To: } \\ 2014 & 2018\end{array}$

Figura 4: Búsqueda Avanzada en rraae

Los resultados obtenidos se muestran en la Figura 5:

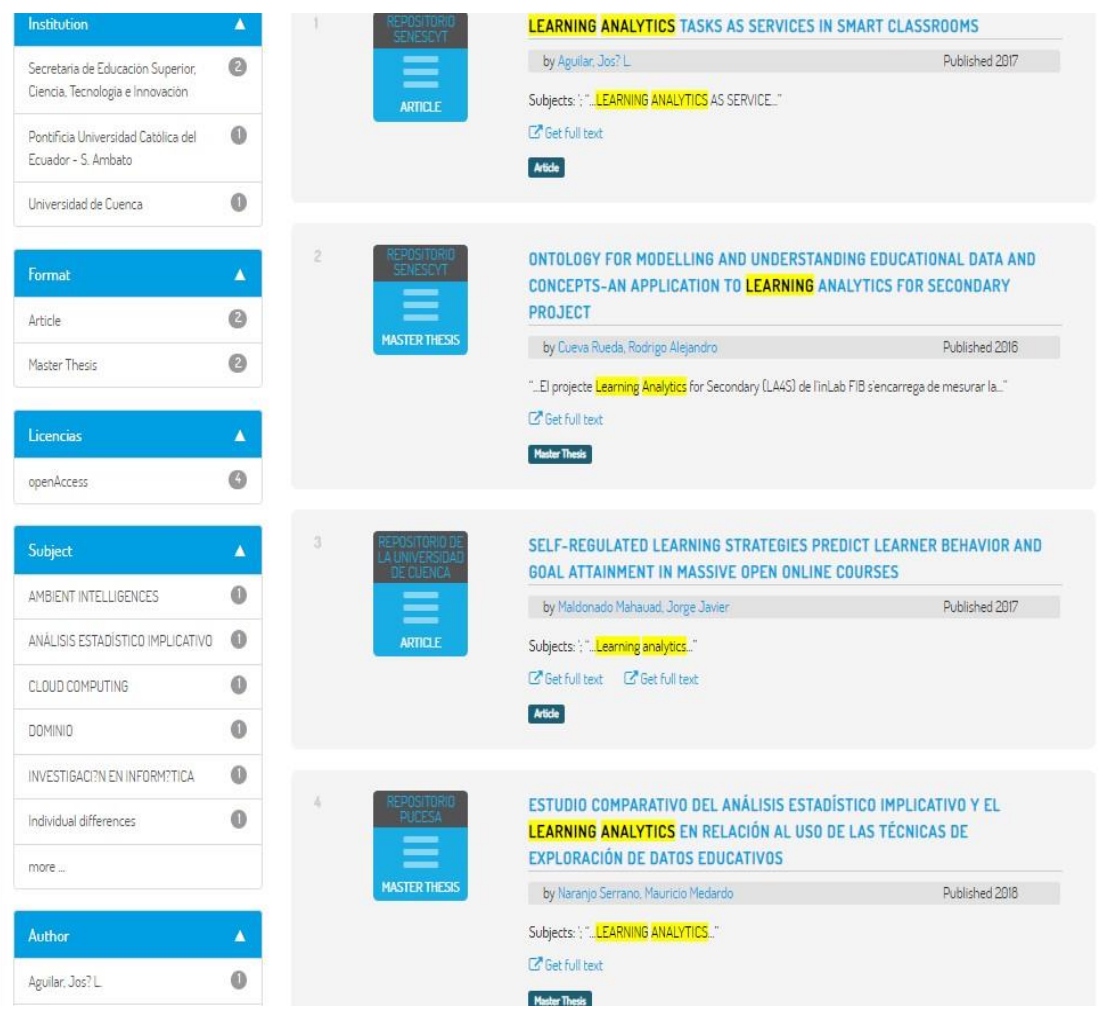

Figura 5: Resultados de la búsqueda Avanzada en rraae 
En la Figura 6, se muestra las opciones de búsqueda para encontrar todos los documentos sobre Analíticas de Aprendizaje que se encuentran en el repositorio digital de la Secretaría de Educación Superior, Ciencia, Tecnología e Innovación (Senescyt)

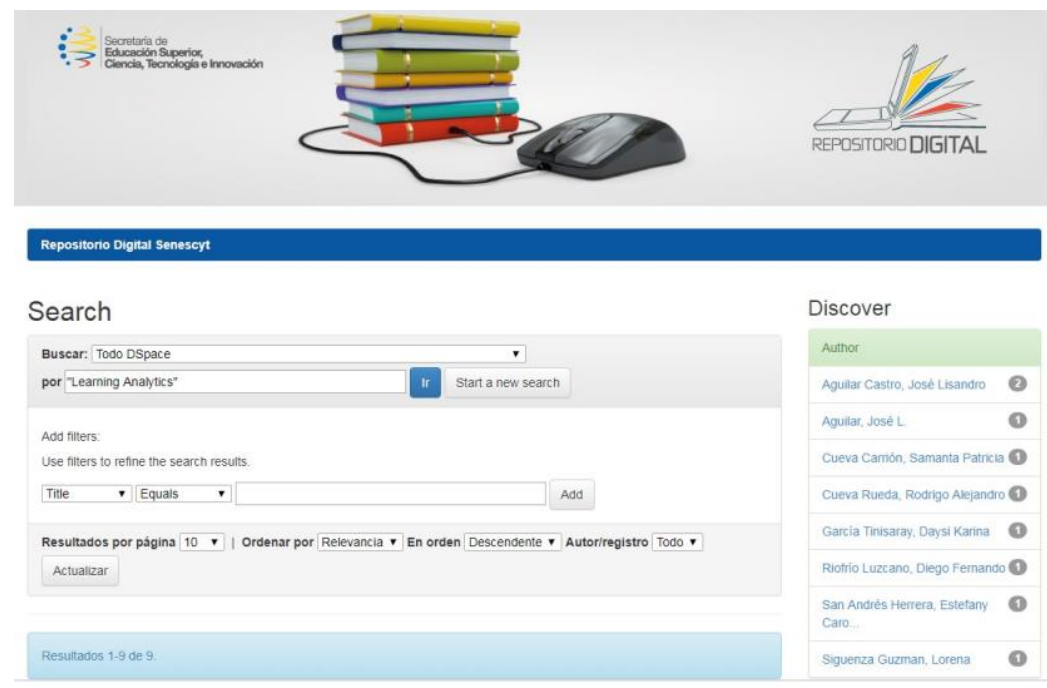

Figura 6: Búsqueda Avanzada en el Repositorio Digital Senescyt

En la Figura 7, se puede observar los nueve documentos relacionados con las Analíticas de Aprendizaje y que reposan en el Repositorio Digital Senescyt.

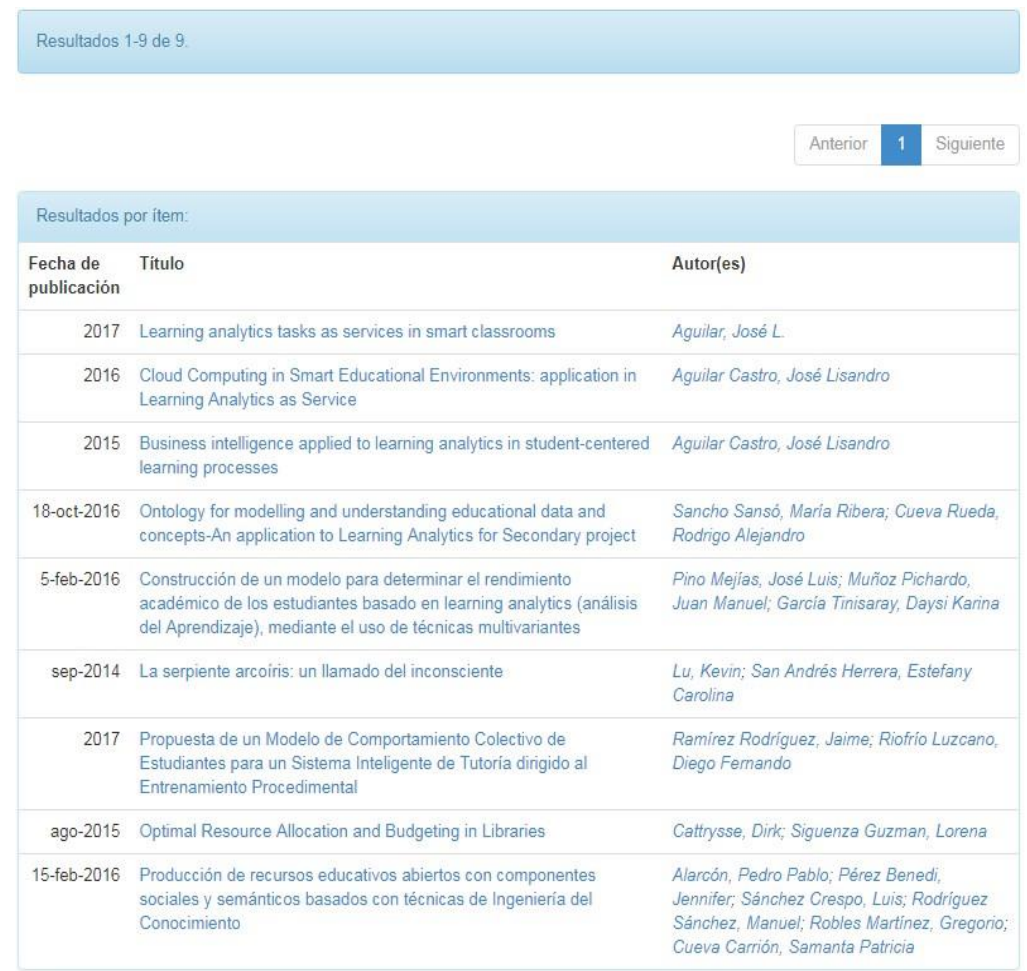

Figura 7: Documentos encontrados en la Búsqueda Avanzada del Repositorio Digital Senescyt 
Los resultados obtenidos se muestran en la Tabla 5.

Tabla 5: Total de Resultados obtenidos en las búsquedas

\begin{tabular}{|c|c|c|c|}
\hline No. & Repositorio & Título & Autor(es) \\
\hline 1 & rraae & Learning Analytics Tasks As Services In Smart Classrooms & Aguilar, Jos? L. \\
\hline 2 & rraae & $\begin{array}{l}\text { Ontology For Modelling And Understanding Educational Data } \\
\text { And Concepts-An Application To Learning Analytics For } \\
\text { Secondary Project }\end{array}$ & $\begin{array}{l}\text { Cueva Rueda, Rodrigo } \\
\text { Alejandro; Sancho Sans?, Mar?a } \\
\text { Ribera }\end{array}$ \\
\hline 3 & rraae & $\begin{array}{l}\text { Self-Regulated Learning Strategies Predict Learner Behavior } \\
\text { And Goal Attainment In Massive Open Online Courses }\end{array}$ & $\begin{array}{l}\text { Maldonado Mahauad, Jorge } \\
\text { Javier }\end{array}$ \\
\hline 4 & rraae & $\begin{array}{l}\text { Estudio Comparativo Del Análisis Estadístico Implicativo Y } \\
\text { El Learning Analytics En Relación Al Uso De Las Técnicas } \\
\text { De Exploración De Datos Educativos }\end{array}$ & $\begin{array}{l}\text { Naranjo Serrano, Mauricio } \\
\text { Medardo }\end{array}$ \\
\hline 5 & Senescyt & $\begin{array}{l}\text { Cloud Computing in Smart Educational Environments: } \\
\text { application in Learning Analytics as Service }\end{array}$ & Aguilar Castro, José Lisandro \\
\hline 6 & Senescyt & $\begin{array}{l}\text { Business intelligence applied to learning analytics in student- } \\
\text { centered learning processes }\end{array}$ & Aguilar Castro, José Lisandro \\
\hline 7 & Senescyt & $\begin{array}{l}\text { Construcción de un modelo para determinar el rendimiento } \\
\text { académico de los estudiantes basado en learning analytics } \\
\text { (análisis del Aprendizaje), mediante el uso de técnicas } \\
\text { multivariantes }\end{array}$ & $\begin{array}{l}\text { Pino Mejías, José Luis; Muñoz } \\
\text { Pichardo, Juan Manuel; García } \\
\text { Tinisaray, Daysi Karina }\end{array}$ \\
\hline 8 & Senescyt & Learning analytics tasks as services in smart classrooms & Aguilar, José L. \\
\hline 9 & Senescyt & $\begin{array}{l}\text { Ontology for modelling and understanding educational data } \\
\text { and concepts-An application to Learning Analytics for } \\
\text { Secondary project }\end{array}$ & $\begin{array}{l}\text { Sancho Sansó, María } \\
\text { Ribera; Cueva Rueda, Rodrigo } \\
\text { Alejandro }\end{array}$ \\
\hline 10 & Senescyt & La serpiente arcoíris: un llamado del inconsciente & $\begin{array}{l}\text { Lu, Kevin; San Andrés Herrera, } \\
\text { Estefany Carolina }\end{array}$ \\
\hline 11 & Senescyt & $\begin{array}{l}\text { Propuesta de un Modelo de Comportamiento Colectivo de } \\
\text { Estudiantes para un Sistema Inteligente de Tutoría dirigido al } \\
\text { Entrenamiento Procedimental }\end{array}$ & $\begin{array}{l}\text { Ramírez Rodríguez, } \\
\text { Jaime; Riofrío Luzcano, Diego } \\
\text { Fernando }\end{array}$ \\
\hline 12 & Senescyt & Optimal Resource Allocation and Budgeting in Libraries & $\begin{array}{l}\text { Cattrysse, Dirk; Siguenza } \\
\text { Guzman, Lorena }\end{array}$ \\
\hline 13 & Senescyt & $\begin{array}{l}\text { Producción de recursos educativos abiertos con componentes } \\
\text { sociales y semánticos basados con técnicas de Ingeniería del } \\
\text { Conocimiento }\end{array}$ & $\begin{array}{l}\text { Alarcón, Pedro Pablo; Pérez } \\
\text { Benedi, Jennifer; Sánchez } \\
\text { Crespo, Luis; Rodríguez } \\
\text { Sánchez, Manuel; Robles } \\
\text { Martínez, Gregorio; Cueva } \\
\text { Carrión, Samanta Patricia }\end{array}$ \\
\hline
\end{tabular}

Los criterios de calidad se basan sobre todo en que el origen de los documentos garantice estatalmente que son de Universidades Ecuatorianas, en este caso al trabajar con Fuentes como el rraae y Senescyt, se logra que en todos los documentos obtenidos el criterio de calidad se cumpla. 
Los criterios de Inclusión utilizados fueron de que los documentos pertenecieran al período 2014-2018, en este caso todos los documentos cumplen y por tanto se mantienen. No hay documentos duplicados entonces se mantienen los 13 documentos. Además, que los documento traten de Analices de Aprendizaje (o Learning Analytics) obteniéndose que únicamente 7 documentos cumplen este criterio, la Tabla 6, muestran estos resultados.

Tabla 6: Total de Resultados aplicando los criterios de inclusión

\begin{tabular}{|c|c|c|}
\hline Repositorio & Título & Autor(es) \\
\hline rraae & $\begin{array}{l}\text { Learning Analytics Tasks As Services In Smart } \\
\text { Classrooms }\end{array}$ & Aguilar, Jos? L. \\
\hline rraae & $\begin{array}{l}\text { Ontology For Modelling And Understanding } \\
\text { Educational Data And Concepts-An Application To } \\
\text { Learning Analytics For Secondary Project }\end{array}$ & $\begin{array}{l}\text { Cueva Rueda, } \\
\text { Rodrigo Alejandro; } \\
\text { Sancho Sans?, Mar?a } \\
\text { Ribera }\end{array}$ \\
\hline rraae & $\begin{array}{l}\text { Self-Regulated Learning Strategies Predict Learner } \\
\text { Behavior And Goal Attainment In Massive Open } \\
\text { Online Courses }\end{array}$ & $\begin{array}{l}\text { Maldonado Mahauad, } \\
\text { Jorge Javier }\end{array}$ \\
\hline rraae & $\begin{array}{l}\text { Estudio Comparativo Del Análisis Estadístico } \\
\text { Implicativo Y El Learning Analytics En Relación Al } \\
\text { Uso De Las Técnicas De Exploración De Datos } \\
\text { Educativos }\end{array}$ & $\begin{array}{l}\text { Naranjo Serrano, } \\
\text { Mauricio Medardo }\end{array}$ \\
\hline Senescyt & $\begin{array}{l}\text { Cloud Computing in Smart Educational } \\
\text { Environments: application in Learning Analytics as } \\
\text { Service }\end{array}$ & $\begin{array}{l}\text { Aguilar Castro, José } \\
\text { Lisandro }\end{array}$ \\
\hline Senescyt & $\begin{array}{l}\text { Business intelligence applied to learning analytics in } \\
\text { student-centered learning processes }\end{array}$ & $\begin{array}{l}\text { Aguilar Castro, José } \\
\text { Lisandro }\end{array}$ \\
\hline Senescyt & $\begin{array}{l}\text { Construcción de un modelo para determinar el } \\
\text { rendimiento académico de los estudiantes basado en } \\
\text { learning analytics (análisis del Aprendizaje), } \\
\text { mediante el uso de técnicas multivariantes }\end{array}$ & $\begin{array}{l}\text { Pino Mejías, José } \\
\text { Luis; Muñoz } \\
\text { Pichardo, Juan } \\
\text { Manuel; García } \\
\text { Tinisaray, Daysi } \\
\text { Karina }\end{array}$ \\
\hline
\end{tabular}


Luego de realizar un tamizado con los 7 documentos restantes, se aplicó el criterio de exclusión que deben ser Trabajos de Graduación y se obtuvieron el número final de 3 documentos que se muestran en la Tabla 7.

Tabla 7: Total de Resultados aplicando los criterios de exclusión

\begin{tabular}{|c|c|c|c|}
\hline Repositorio & Título & Autor(es) & Cita \\
\hline rraae & $\begin{array}{l}\text { Ontology For Modelling And } \\
\text { Understanding Educational Data And } \\
\text { Concepts-An Application To Learning } \\
\text { Analytics For Secondary Project }\end{array}$ & $\begin{array}{l}\text { Cueva Rueda, } \\
\text { Rodrigo } \\
\text { Alejandro; } \\
\text { Sancho Sans?, } \\
\text { Mar?a Ribera }\end{array}$ & $\begin{array}{l}\text { (Casanovas } \\
\text { Muñoz, 2016) }\end{array}$ \\
\hline rraae & $\begin{array}{l}\text { Estudio Comparativo Del Análisis } \\
\text { Estadístico Implicativo Y El Learning } \\
\text { Analytics En Relación Al Uso De Las } \\
\text { Técnicas De Exploración De Datos } \\
\text { Educativos }\end{array}$ & $\begin{array}{l}\text { Naranjo Serrano, } \\
\text { Mauricio } \\
\text { Medardo }\end{array}$ & $\begin{array}{l}\text { (Naranjo } \\
\text { Serrano, 2018) }\end{array}$ \\
\hline Senescyt & $\begin{array}{l}\text { Construcción de un modelo para } \\
\text { determinar el rendimiento académico de } \\
\text { los estudiantes basado en learning } \\
\text { analytics (análisis del Aprendizaje), } \\
\text { mediante el uso de técnicas } \\
\text { multivariantes }\end{array}$ & $\begin{array}{l}\text { Pino Mejías, } \\
\text { José } \\
\text { Luis; Muñoz } \\
\text { Pichardo, Juan } \\
\text { Manuel; García } \\
\text { Tinisaray, Daysi } \\
\text { Karina }\end{array}$ & $\begin{array}{c}\text { (Tinisaray \& } \\
\text { Karina, 2016) }\end{array}$ \\
\hline
\end{tabular}

A continuación, se procede a responder las preguntas de investigación planteadas, para hacerlas parte de las conclusiones.

\section{Resultados}

En esta sección se respondieron todas las preguntas de investigación planteadas.

RQ1: ¿Cuáles Instituciones de Educación Superior tienen Trabajos de Graduación sobre Analíticas de Aprendizaje?

La Tabla 8, muestra las Instituciones de Educación Superior responsables de los Trabajos de Graduación sobre Analíticas de Aprendizaje 
Tabla 8: IES con Trabajos de Graduación sobre Analíticas de Aprendizaje

\begin{tabular}{|c|c|c|}
\hline IES & Título & Autor(es) \\
\hline $\begin{array}{l}\text { Senescyt: } \\
\text { Universidad } \\
\text { Politécnica de Cataluña }\end{array}$ & $\begin{array}{l}\text { Ontology For Modelling And } \\
\text { Understanding Educational Data And } \\
\text { Concepts-An Application To Learning } \\
\text { Analytics For Secondary Project }\end{array}$ & $\begin{array}{l}\text { Cueva Rueda, } \\
\text { Rodrigo } \\
\text { Alejandro; } \\
\text { Sancho Sans?, } \\
\text { Mar?a Ribera }\end{array}$ \\
\hline $\begin{array}{l}\text { Pontificia Universidad } \\
\text { Católica del Ecuador- } \\
\text { Sede Ambato }\end{array}$ & $\begin{array}{l}\text { Estudio Comparativo Del Análisis } \\
\text { Estadístico Implicativo Y El Learning } \\
\text { Analytics En Relación Al Uso De Las } \\
\text { Técnicas De Exploración De Datos } \\
\text { Educativos }\end{array}$ & $\begin{array}{l}\text { Naranjo Serrano, } \\
\text { Mauricio } \\
\text { Medardo }\end{array}$ \\
\hline $\begin{array}{l}\text { Senescyt: } \\
\text { Universidad de Sevilla/ } \\
\text { Universidad Técnica } \\
\text { Particular de Loja }\end{array}$ & $\begin{array}{l}\text { Construcción de un modelo para } \\
\text { determinar el rendimiento académico de } \\
\text { los estudiantes basado en learning } \\
\text { analytics (análisis del Aprendizaje), } \\
\text { mediante el uso de técnicas multivariantes }\end{array}$ & $\begin{array}{l}\text { Pino Mejías, José } \\
\text { Luis; Muñoz } \\
\text { Pichardo, Juan } \\
\text { Manuel; García } \\
\text { Tinisaray, Daysi } \\
\text { Karina }\end{array}$ \\
\hline
\end{tabular}

RQ2: ¿Cuáles Trabajos de Graduación sobre Analíticas de Aprendizaje son de pregrado o postgrado?

La Tabla 9, muestra los Tipos de Trabajos de Graduación ecuatorianos sobre Analíticas de Aprendizaje.

Tabla 9: Tipos de Trabajos de Graduación ecuatorianos

\begin{tabular}{cll}
\hline Tipo & \multicolumn{1}{c}{ Título } & \multicolumn{1}{c}{ Autor(es) } \\
\hline Master & Ontology For Modelling And Understanding & Cueva Rueda, Rodrigo \\
Thesis & $\begin{array}{l}\text { Educational Data And Concepts-An Application } \\
\text { To Learning Analytics For Secondary Project }\end{array}$ & $\begin{array}{l}\text { Alejandro; Sancho } \\
\text { Sans?, Mar?a Ribera }\end{array}$ \\
& Estudio Comparativo Del Análisis Estadístico & \\
Master & Implicativo Y El Learning Analytics En & Naranjo Serrano, \\
Thesis & Relación Al Uso De Las Técnicas De & Mauricio Medardo \\
& Exploración De Datos Educativos & \\
Doctoral & Construcción de un modelo para determinar el & Pino Mejías, José \\
Thesis & rendimiento académico de los estudiantes basado & Luis; Muñoz Pichardo, \\
& &
\end{tabular}


en learning analytics (análisis del Aprendizaje), Juan Manuel; García mediante el uso de técnicas multivariantes

Tinisaray, Daysi Karina

RQ3: ¿Cuáles son las principales palabras clave de los Trabajos de Graduación sobre Analíticas de Aprendizaje en Instituciones de Educación Superior Ecuatorianas?

La Tabla 10, muestra las palabras clave por cada uno de los Trabajos de Titulación, el último Trabajo no muestra ninguna palabra clave.

Tabla 10: Palabras clave en los Tipos de Trabajos de Graduación ecuatorianos

\begin{tabular}{|c|c|c|}
\hline Título & Keywords & Autor(es) \\
\hline $\begin{array}{l}\text { Ontology For Modelling And } \\
\text { Understanding Educational Data And } \\
\text { Concepts-An Application To Learning } \\
\text { Analytics For Secondary Project }\end{array}$ & $\begin{array}{l}\text { Investigación En Informática, } \\
\text { Ontología, Paradigma, Owl, } \\
\text { Obda, Semántica, Uml, Lms, } \\
\text { Dominio, Linked Data }\end{array}$ & $\begin{array}{l}\text { Cueva Rueda, } \\
\text { Rodrigo } \\
\text { Alejandro; } \\
\text { Sancho Sans?, } \\
\text { Mar?a Ribera }\end{array}$ \\
\hline $\begin{array}{l}\text { Estudio Comparativo Del Análisis } \\
\text { Estadístico Implicativo Y El Learning } \\
\text { Analytics En Relación Al Uso De Las } \\
\text { Técnicas De Exploración De Datos } \\
\text { Educativos }\end{array}$ & $\begin{array}{l}\text { Learning Analytics, Análisis } \\
\text { Estadístico Implicativo, } \\
\text { Métodos Cluster, Minería De } \\
\text { Reglas De Asociación }\end{array}$ & $\begin{array}{l}\text { Naranjo } \\
\text { Serrano, } \\
\text { Mauricio } \\
\text { Medardo }\end{array}$ \\
\hline $\begin{array}{l}\text { Construcción De Un Modelo Para } \\
\text { Determinar El Rendimiento Académico } \\
\text { De Los Estudiantes Basado En } \\
\text { Learning Analytics (Análisis Del } \\
\text { Aprendizaje), Mediante El Uso De } \\
\text { Técnicas Multivariantes }\end{array}$ & No existen palabras clave & $\begin{array}{l}\text { Pino Mejías, } \\
\text { José } \\
\text { Luis; Muñoz } \\
\text { Pichardo, Juan } \\
\text { Manuel; García } \\
\text { Tinisaray, } \\
\text { Daysi Karina }\end{array}$ \\
\hline
\end{tabular}

RQ4: ¿Cuáles son los años de publicación y tendencia de los Trabajos de graduación sobre Analíticas de Aprendizaje en Instituciones de Educación Superior Ecuatorianas?

La Tabla 11, muestra los años de publicación por cada uno de los Trabajos de Graduación. 
Tabla 11: Años de publicación de los Trabajos de Graduación

\begin{tabular}{cll}
\hline $\begin{array}{c}\text { Fecha de } \\
\text { publicación }\end{array}$ & \multicolumn{1}{c}{ Título } & \multicolumn{1}{c}{ Autor(es) } \\
\hline \multirow{2}{*}{2016} & Ontology For Modelling And & Cueva Rueda, \\
& Understanding Educational Data And & \multicolumn{1}{c}{ Rodrigo } \\
& Concepts-An Application To Learning & Alejand Sancho \\
& Analytics For Secondary Project & Sans?, Mar?a \\
& & Ribera \\
\multirow{2}{*}{2016} & Construcción De Un Modelo Para & Pino Mejías, José \\
& Determinar El Rendimiento Académico De & Luis; Muñoz \\
& Los Estudiantes Basado En Learning & Pichardo, Juan \\
& Analytics (Análisis Del Aprendizaje), & Manuel; García \\
& Mediante El Uso De Técnicas & Tinisaray, Daysi \\
& Multivariantes & Karina \\
& & \\
& Estudio Comparativo Del Análisis & \\
& Estadístico Implicativo Y El Learning & Naranjo Serrano, \\
2018 & Analytics En Relación Al Uso De Las & Mauricio Medardo \\
& Técnicas De Exploración De Datos & \\
& Educativos & \\
& &
\end{tabular}

La Figura 8, muestra la línea de tendencia en los Trabajos de Graduación considerando los dos puntos de datos históricos.

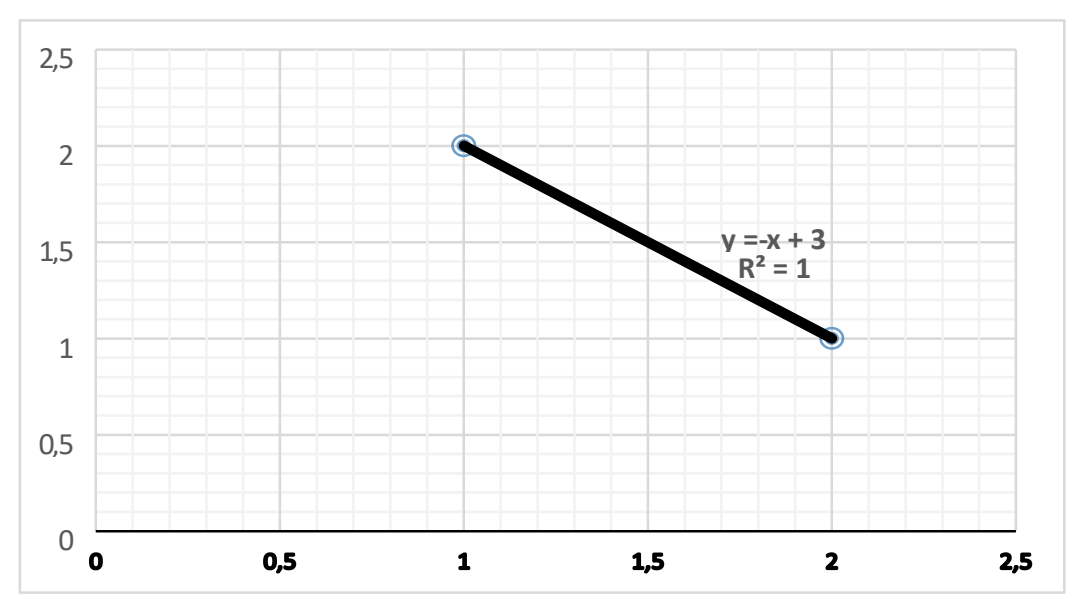

Figura 8: Tendencia del número de Trabajos de Graduación 
RQ5: ¿Cuáles son los autores y coautores de los Trabajos de Graduación sobre Analíticas de Aprendizaje en Instituciones de Educación Superior Ecuatorianas?

La Tabla 12, muestra los autores y coautores por cada uno de los Trabajos de Graduación.

Tabla 12: Años de publicación de los Trabajos de Graduación

\begin{tabular}{lll}
\hline \multicolumn{1}{c}{ Título } & Autor(es) & \multicolumn{1}{c}{ Coautor(es) } \\
\hline $\begin{array}{l}\text { Ontology For Modelling And Understanding } \\
\text { Educational Data And Concepts-An Application } \\
\text { To Learning Analytics For Secondary Project }\end{array}$ & $\begin{array}{l}\text { Jordi } \\
\text { Casanovas } \\
\text { Muñoz }\end{array}$ & $\begin{array}{l}\text { Maria Ribera } \\
\text { Sancho, Albert } \\
\text { Obiols }\end{array}$ \\
$\begin{array}{l}\text { Construcción De Un Modelo Para Determinar El } \\
\text { Rendimiento Académico De Los Estudiantes }\end{array}$ & Daysi & José Luis Pino \\
Basado En Learning Analytics (Análisis Del & Karina & Mejías, Juan \\
$\begin{array}{l}\text { Aprendizaje), Mediante El Uso De Técnicas } \\
\text { Multivariantes }\end{array}$ & García & Manuel Muñoz \\
Estudio Comparativo Del Análisis Estadístico & Naranjo & Pichardo \\
Implicativo Y El Learning Analytics En Relación & Serrano, & Pazmiño Maji, \\
Al Uso De Las Técnicas De Exploración De & Mauricio & Rubén Antonio \\
Datos Educativos & Medardo & \\
& &
\end{tabular}

A continuación, se muestran las conclusiones basadas en los resultados obtenidos.

\section{Conclusiones}

- Una de las Universidades es la Pontificia Universidad Católica del Ecuador con Sede en la Ciudad de Ambato, otras dos tesis fueron realizadas por becarios ecuatorianos del Senescyt: Universidad de Sevilla/ Universidad Técnica Particular de Loja y Universidad Politécnica de Cataluña (Ver Tabla 8).

- No hay ningún trabajo de graduación de pregrado sobre Learning Analytics. Hay tres trabajos de graduación de postgrado 2 de maestría y uno de Doctorado (Ver Tabla 9).

- Las palabras clase se muestran a continuación: Ontología, Paradigma, Owl, Obda, Semántica, Uml, Lms, Dominio, Linked Data, Análisis Estadístico Implicativo, Métodos Cluster, Minería De Reglas De Asociación. No se muestran palabras clave más frecuentes comunes (Ver Tabla 10).

- El año de publicación más frecuente es el 2016 con dos Trabajos de Graduación, el documento más reciente es del 2018 titulado: "estudio comparativo del análisis estadístico implicativo y el learning analytics en relación al uso de las técnicas de exploración de datos educativos" (Ver Tabla 11). La tendencia está dada por la recta de regresión $y=x+3$ (Ver figura 8). 
- Los autores principales son las personas que elaboraron los Trabajos de Graduación (Jordi Casanovas Muñoz, Daysi Karina García Tinisaray, Naranjo Serrano Mauricio Medardo), los coautores con los directores de los trabajos de titulación (Maria Ribera Sancho, Albert Obiols; José Luis Pino Mejías, Juan Manuel Muñoz Pichardo, Pazmiño Maji, Rubén Antonio) (Ver Tabla 12).

Se recomienda continuar este estudio y extenderlo para profundizar los Trabajos de Graduación encontrados.

\section{Referencias Bibliográficas}

Buscar en Gaceta Oficial | CES - Consejo de Educación Superior | Ecuador. (s. f.). $\begin{array}{llllll}\text { Recuperado } & 29 & \text { de } & \text { julio }\end{array}$ http://www.ces.gob.ec/index.php?option=com_sobipro\&task=search\&sid=741\&Ite $\operatorname{mid}=0$

Casanovas Muñoz, J. (2016). Ontology for modelling and understanding educational data and concepts: An application to Learning Analytics for Secondary project. Universitat Politècnica de Catalunya.

CES - Consejo de Educación Superior | Ecuador. (s. f.). Recuperado 29 de julio de 2019, de http://www.ces.gob.ec/

Coleman, J. (s. f.). Subject Guides: Systematic Reviews - Research Guide: Defining Your Review Question. Recuperado 29 de julio de 2019, de https://libguides.murdoch.edu.au/systematic/defining

Dos Santos, H. L., Cechinel, C., Nunes, J. B. C., \& Ochoa, X. (2017). An initial review of learning analytics in Latin America. 2017 Twelfth Latin American Conference on Learning Technologies (LACLO), 1-9. IEEE.

DSpace: An Open Source Dynamic Digital Repository. (s. f.). Recuperado 29 de julio de 2019, de http://www.dlib.org/dlib/january03/smith/01smith.html

Estadísticas. (s. f.). Recuperado 1 de agosto de 2019, de http://www.rraae.org.ec/Statistics/Map

Feliz González, D. (2017). La sociedad del futuro: Las tecnologías ubicuas y su impacto en la sociedad.

Freile, C. (2015). Hitos de la historia de la educación en el Ecuador. Recuperado el.

Hausner, E., \& Waffenschmidt, S. (2016). Development of search strategies for systematic reviews: Reply to commentary by Dintsios and Niederstadt (Letter commenting on: J Clin Epidemiol. 2015; 68: 191-199). Journal of clinical epidemiology, 69, 265. 
Jesson, J., Matheson, L., \& Lacey, F. M. (2011). Doing your literature review. Los Angeles Calif.; London: SAGE.

Johnson, L., Becker, S. A., Cummins, M., Estrada, V., Freeman, A., \& Hall, C. (2016).

NMC horizon report: 2016 higher education edition. The New Media Consortium.

Kitchenham, B. A., Budgen, D., \& Brereton, P. (2016). Evidence-based software engineering and systematic reviews. Recuperado de http://proquest.tech.safaribooksonline.de/9781482228663

Methley, A. M., Campbell, S., Chew-Graham, C., McNally, R., \& Cheraghi-Sohi, S. (2014). PICO, PICOS and SPIDER: a comparison study of specificity and sensitivity in three search tools for qualitative systematic reviews. BMC health services research, 14(1), 579.

Naranjo Serrano, M. M. (2018). Estudio comparativo del análisis estadístico implicativo y el learning analytics en relación al uso de las técnicas de exploración de datos educativos. Pontificia Universidad Católica del Ecuador.

Ochoa, X. (2019). Learning analytics in Latin America present an opportunity not to be missed. Nature human behaviour, 3(1), 6 .

Pazmiño-Maji, R. A., García-Peñalvo, F. J., \& Conde-González, M. A. (2017). Mapeo Sistemático en B-Learning: Aplicación a cursos moodle sobre Análisis Estadístico Implicativo.

Pazmiño-Maji, R. A., García-Peñalvo, F. J., \& Conde-González, M. A. (s. f.). Approximation of statistical implicative analysis to learning analytics. 355-376.

Pazmiño-Maji, R. A., Solis-Benavides, C. S., García-Peñalvo, F. J., \& Conde-González, M. A. (2019). LA INVESTIGACION DE PREGRADO EN LA ESCUELA SUPERIOR POLITECNICA DE CHIMBORAZO: MAPEO SISTEMATICO Y ANALITICAS. Revista Científica ECOCIENCIA, 6, 25. Recuperado de http://ecociencia.ecotec.edu.ec/upload/php/files/febrero19/02.pdf

Pazmiño-Maji, R. A., Valverde-Aguirre, P. E., García-Peñalvo, F. J., \& Conde-González, M. A. (2019). Caracterización de la investigación de pregrado en la Escuela Superior Politécnica de Chimborazo: Un Mapeo Sistemático con nuevas técnicas de las Analíticas de Aprendizaje. VII evento internacional La Universidad En El Siglo XXI.

Portal Ciudadano SNIESE. (s. f.). Recuperado 30 de julio de 2019, de https://infoeducacionsuperior.gob.ec/\#/ies-acreditadas

Repositorio Digital Senescyt: Página de inicio. (s. f.). Recuperado 31 de julio de 2019, de http://repositorio.educacionsuperior.gob.ec/

Rraae. (s. f.). Recuperado 29 de julio de 2019, de http://www.rraae.org.ec/

Siemens, G., \& Long, P. (2011). Penetrating the fog: Analytics in learning and education. EDUCAUSE review, 46(5), 30. 
Tinisaray, G., \& Karina, D. (2016). Construcción de un modelo para determinar el rendimiento académico de los estudiantes basado en learning analytics (análisis del aprendizaje), mediante el uso de técnicas multivariantes.

\section{Para citar el artículo indexado.}

Pazmiño Maji, R., López Ortega, J., Conde González, M. \& García Peñalvo, F. (2019). Las analíticas de aprendizaje en el Ecuador: Un análisis inicial basado en el mapeo sistemático de los trabajos de graduación. Explorador Digital, 3(3.1), 224-245. https://doi.org/10.33262/exploradordigital.v3i3.1.885

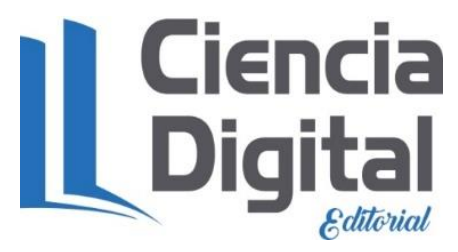

El artículo que se publica es de exclusiva responsabilidad de los autores y no necesariamente reflejan el pensamiento de la Revista Explorador Digital.

El articulo queda en propiedad de la revista y, por tanto, su publicación parcial y/o total en otro medio tiene que ser autorizado por el director o editor de la Revista Explorador Digital.
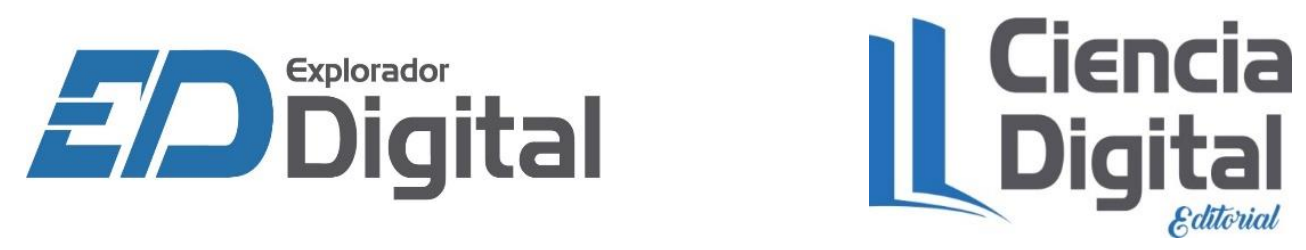